\title{
海藻酸钠离子凝胶法制备直通孔氧化铝多孔陶瓷
}

\author{
孙 阳 ${ }^{1,2}$, 薛伟江 ${ }^{2}$, 孙加林 ${ }^{1}$, 周国治 ${ }^{1}$, 黄 勇 $^{2}$
}

(1. 北京科技大学 材料科学与工程学院, 北京 $100083 ; 2$. 清华大学 新型陶瓷与精细工艺国家重点实验室, 材料科 学与工程学院, 北京 100084)

摘 要: 利用海藻酸钠的离子凝胶过程, 采用溶剂置换结合冷冻干燥的工艺, 成功制备了具有高度有序六方排列 的直通孔多孔氧化铝陶瓷，整个工艺过程及所使用的原料都是环境友好的。研究结果表明, $1500^{\circ} \mathrm{C}$ 烧结 $2 \mathrm{~h}$ 样品 的孔径尺寸在 $200 \mu \mathrm{m}$ 左右，且与固相含量的关系不大，而孔壁上存在 $0.3 \mu \mathrm{m} \sim 0.5 \mu \mathrm{m}$ 的小孔。通过控制浆料中 氧化铝的固相含量可以对材料的性能进行有效地调控, 研究表明, 随着固相含量从 $5 \mathrm{wt} \%$ 提高到 $15 \mathrm{wt} \%$, 材料的密 度从 $0.87 \mathrm{~g} / \mathrm{cm}^{3}$ 提高到 $1.16 \mathrm{~g} / \mathrm{cm}^{3}$, 渗透率从 $2.57 \times 10^{-11} \mathrm{~m}^{2}$ 下降到 $2.16 \times 10^{-11} \mathrm{~m}^{2}$, 而抗压强度从(18.9土3.2) MPa 提 高到(44.2 \pm 5.4$) \mathrm{MPa}$, 平行孔道方向的热导率从 $2.1 \mathrm{~W} /(\mathrm{m} \cdot \mathrm{K})$ 提高到 $3.1 \mathrm{~W} /(\mathrm{m} \cdot \mathrm{K})$, 而垂直孔道方向的热导率从 $1.3 \mathrm{~W} /(\mathrm{m} \cdot \mathrm{K})$ 提高到 $1.7 \mathrm{~W} /(\mathrm{m} \cdot \mathrm{K})$, 并且平行孔道方向热导率的增加幅度要明显大于垂直孔道方向。

关 键 词: 直通孔氧化铝多孔陶瓷; 渗透率; 热导率; 离子凝胶

中图分类号: TQ174 文献标识码: A

\section{Porous Alumina Ceramics with Unidirectional Oriented Pores Fabricated by Ionotropic Process of Sodium Alginate}

\author{
SUN Yang ${ }^{1,2}$, XUE Wei-Jiang ${ }^{2}$, SUN Jia-Lin ${ }^{1}$, ZHOU Guo-Zhi ${ }^{1}$, HUANG Yong ${ }^{2}$
}

(1. School of Material Science and Engineering, University of Science \& Technology Beijing, Beijing 100083, China; 2. State Key Laboratory of New Ceramics and Fine Processing, Department of Materials Science and Engineering, Tsinghua University, Beijing 100084, China)

\begin{abstract}
Alumina ceramic bodies with high porosity characterized by highly ordered and unidirectional oriented pores were successfully fabricated using the ionotropic process of sodium alginate by solvent exchange subsequently with freeze-drying. It is important to point out that the whole process and raw materials are eco-friendly. The average unidirectional pore size of samples sintered at $1500^{\circ} \mathrm{C}$ for $2 \mathrm{~h}$ is $200 \mu \mathrm{m}$ with minor porosity in the pore walls with average pore size of 0.3-0.5 $\mu \mathrm{m}$. The properties of samples can be adjusted by controlling the solid loading in slurry. As the solid loading increasing from $5 \mathrm{wt} \%$ to $15 \mathrm{wt} \%$, the density and compressive strength increased from $0.87 \mathrm{~g} / \mathrm{cm}^{3}$ to $1.16 \mathrm{~g} / \mathrm{cm}^{3}$ and from $(18.9 \pm 3.2) \mathrm{MPa}$ to $(44.2 \pm 5.4) \mathrm{MPa}$, respectively with permeability decreasing from $2.57 \times 10^{-11} \mathrm{~m}^{2}$ to $2.16 \times 10^{-11} \mathrm{~m}^{2}$. In addition, with the solid loading increasing from $5 \mathrm{wt} \%$ to $15 \mathrm{wt} \%$, the conductivity of the direction parallel and perpendicular to the unidirectional pores increased from $2.1 \mathrm{~W} /(\mathrm{m} \cdot \mathrm{K})$ to $3.1 \mathrm{~W} /(\mathrm{m} \cdot \mathrm{K})$ and from $1.3 \mathrm{~W} /(\mathrm{m} \cdot \mathrm{K})$ to $1.7 \mathrm{~W} /(\mathrm{m} \cdot \mathrm{K})$, respectively.
\end{abstract}

Key words: unidirectionally oriented porous alumina ceramics; permeability; thermal conductivity; ionotropic

多孔陶瓷的发展始于 19 世纪 70 年代, 最初用 作铀提纯材料和细菌过滤材料 ${ }^{[1]}$ 。多孔陶瓷具有体

收稿日期: 2015-01-22; 收到修改稿日期：2015-04-21

基金项目：国家重点基础研究发展计划(973 计划)(2006CB605207-2)

National Key Basic Research and Developmet Plan (973Plan)(2006CB605207-2)

作者简介: 孙 阳(1956-), 男, 博士研究生. E-mail: yangsun8@yahoo.com

通讯作者: 黄 勇, 教授. E-mail: hy-dms@mail.tsinghua.edu.cn 
积密度小、孔隙率较高、比表面积大、对液体和 气体介质有选择透过性、能量吸收或阻尼特性等 特点 ${ }^{[2-3]}$, 尤其是直通孔多孔陶瓷, 在气体液体过 滤、净化分离等多方面得到广泛的应用 ${ }^{[4-5]}$ 。传统制 备直通孔多孔陶瓷的方法有冷冻凝胶 ${ }^{[6-7]}$ 、纤维涂覆 氧化铝溶胶 ${ }^{88]}$ 等, 但是所使用的造孔剂或原料往往 具有毒性，或者在排除造孔剂的过程中会产生污 染。此外, 规则排列的孔结构一直是显微结构调控 的目标, 到目前为止, 只有阳极氧化 ${ }^{[9]}$ 和溶胶一凝胶 法 $^{[10]}$ 可以制备多孔陶瓷膜以及纳米管。

50 年前, Thiele 等 ${ }^{[11]}$ 发现将二价或者三价金属 离子缓慢渗透到海藻酸钠溶胶中可以形成高度规则 排列的毛细管结构。这一过程称为海藻酸钠的离子 凝胶过程，它受到金属离子的扩散控制，类似于 Rayleigh-benard 对流过程 ${ }^{[12]}$ 。Dittrich 等 $^{[13]}$ 利用海 藻酸钠的自组装过程成功制备了具有高度规则排列 直通孔的羟基磷灰石组织支架, 为海藻酸钠在多孔 陶瓷研究领域开辟了新的应用。值得一提的是, 海 藻酸钠 ${ }^{[14-15}$ 是从褐藻类的海带或马尾藻中提取的一 种多糖碳水化合物, 常作为原料或者添加剂应用于 食品领域, 是一种纯天然无毒环保的材料。

本研究使用氧化铝作为原料, 利用海藻酸钠的 离子凝胶过程制备了具有高度规则排列的直通孔多 孔陶瓷, 并着重讨论了工艺参数对材料的微观结 构、气孔分布以及气孔率，渗透率和热导率的影响。

\section{1 实验方法}

\section{1 原料}

实验原料采用氧化铝粉: $\alpha-\mathrm{Al}_{2} \mathrm{O}_{3}$, 青岛美铝公 司生产, 型号为 $\mathrm{CT} 3000\left(D_{50} 0.60 \mu \mathrm{m}\right.$ 左右 $)$; 海藻酸 钠 $(\mathrm{NaAlg})$, 国药集团化学试剂有限公司生产, 化学 纯; 氯化钲, 北京现代东方精细化学品有限公司生 产, 分析纯; 叔丁醇, 国药集团化学试剂有限公司 生产, 化学纯。

\section{2 实验过程}

将 $1.5 \mathrm{wt} \%$ 的海藻酸钠溶液和氧化铝粉按照固 相含量 $5 \mathrm{wt} \% \sim 15 \mathrm{wt} \%$ 混合球磨 $4 \mathrm{~h}$, 然后将球磨得到 的浆料倒入 $100 \mathrm{~mL}$ 烧杯中。将浓度为 $1.5 \mathrm{~mol} / \mathrm{L}$ 的 $\mathrm{CaCl}_{2}$ 溶液均匀喷雾到烧杯中的浆料表面, $\mathrm{Ca}^{2+}$ 会随 着重力作用向下渗透, $36 \mathrm{~h}$ 后浆料就会完全固化, 并在其中形成均匀分布的直通孔道。然后将固化后 的湿坏脱模，浸入 $10 \mathrm{wt} \%$ 的葡萄糖内酯溶液中 $12 \mathrm{~h}$ 进行预收缩, 因为葡萄糖酸内酯可以缓慢水解出 $\mathrm{H}^{+}$, 而海藻酸钠凝胶在酸性条件下会将其中多余的水释
放出来，从而使得凝胶收缩。然后将湿坏浸入叔丁 醇中 $12 \mathrm{~h}$ 进行溶剂置换。将溶剂置换后的湿坏放置 于空气中常温缓慢干燥, 或者采用冷冻干燥法进行 干燥(冷冻温度为 $-70^{\circ} \mathrm{C}$ )。

氧化铝陶瓷的烧结温度通常在 $1500^{\circ} \mathrm{C} \sim 1600^{\circ} \mathrm{C}$ 之间, 烧结温度过高会使材料的收缩过大, 有可能 使材料中存在的定向通孔结构收缩过大，甚至破坏 这种孔结构; 而烧结温度低于 $1500^{\circ} \mathrm{C}$, 则无法获得 足够的强度, 所以本实验将干燥得到的坏体在 $1500^{\circ} \mathrm{C}$ 下烧结 $2 \mathrm{~h}$, 从而得到直通孔多孔氧化铝陶瓷。工艺 流程如图 1 所示。

\section{3 性能表征}

采用日本 Olympus 公司生产的 BX50 型光学显 微镜和日本 Jeol Industries 公司生产的 JSM-6460LV 型扫描电子显微镜进行显微结构观察。采用美国 Micromeritics Instrument Corporation 公司生产的 AutoPore VI 9500 压永仪测试孔径分布。采用美国 Quantum Design 公司生产的 PPMS-9 综合物性测量 系统测试热导率, 试样尺寸为 $5 \mathrm{~mm} \times 5 \mathrm{~mm} \times 3 \mathrm{~mm}$ 的 长方体。采用长春可信试验仪器公司生产的 WDW3020 万能试验机测试抗压强度, 试样尺寸为 $\phi 20 \mathrm{~mm} \times 20 \mathrm{~mm}$ 。渗透率测试: 试样加工成直径> $20 \mathrm{~mm}$, 厚度 $\geqslant 10 \mathrm{~mm}$ (厚度方向为定向排列的孔道 方向)的圆片状进行渗透率测试, 测试仪器为湖南

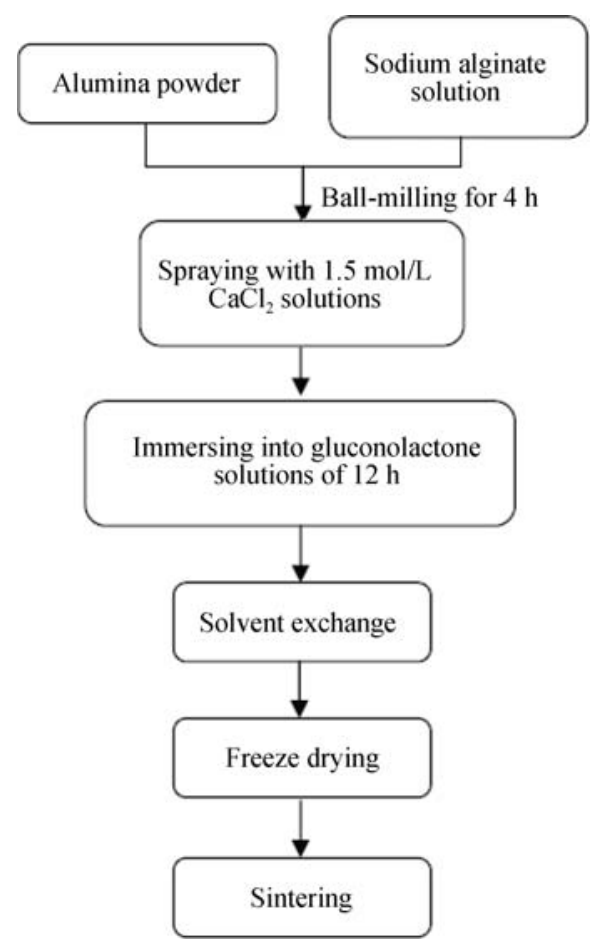

图 1 海藻酸钠离子凝胶法工艺流程图

Fig. 1 Schematic illustrations for fabricating alumina ceramics by sodium alginate ionotropic 
湘潭湘仪器有限公司的 DSY 多孔陶瓷渗透率试验 仪。利用 Darcy 公式 ${ }^{[16]}$ 对测试数据进行计算获得材 料的渗透率:

$$
\Delta P=\eta L Q / \mu A
$$

其中: $\Delta P$ 一样品两侧的压力差, $\mathrm{Pa}$;

$Q$ 一流量, $\mathrm{m}^{3} / \mathrm{s}$;

$\eta$ 一测试用液体(水)粘度, 取 $1.01 \times 10^{-3} \mathrm{~Pa} \cdot \mathrm{s}$;

$A$ 一样品的横截面积, $\mathrm{m}^{2}$;

$L$ 一样品厚度, $\mathrm{m}$;

$\mu$ 一渗透率, $\mathrm{m}^{2}$

\section{2 结果与讨论}

\section{1 直通孔结构的形成}

将金属离子 $\mathrm{Me}^{2+}$ 喷雾于浆料表面后，金属离子 $\mathrm{Me}^{2+}$ 会使海藻酸钠发生聚合, 从而形成一层初始薄 膜, 这层膜结构比较致密, 只允许阳离子透过。初始 薄膜下一定厚度内孔隙结构分布不均匀, 这层结构 的厚度占整个湿坏厚度的比例很小。随着 $\mathrm{Me}^{2+}$ 的继 续渗透, 形成了分布均匀的毛细管结构, 图 2 为海 藻酸钠离子凝胶法制备的样品湿坏结构示意图。 Dittrich 等 ${ }^{[13]}$ 制备的湿坏中, 均匀的直通毛细管孔 道长度达到了 $15 \mathrm{~mm}$ 。当样品厚度达到一定程度, 孔道分布变得不均匀, 孔径增大, 直到孔道结构完 全消失。

\section{2 显微结构}

图 3 为海藻酸钠离子凝胶显微结构的光学显 微镜照片。从图 3(a)中可以看出, 凝胶中的孔呈六 方高度规则排列; 而从图 3(b)中可以看出, 沿着 $\mathrm{Ca}^{2+}$ 的扩散方向, 凝胶中形成了定向排列的孔道 结构。

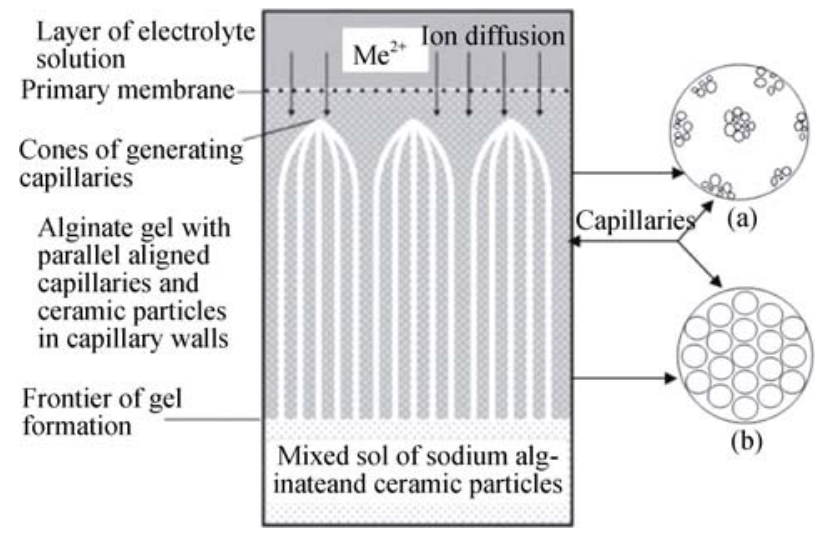

图 2 样品中直通孔结构的形成示意图 ${ }^{[17]}$

Fig. 2 Formation of unidirectional porous structures in samples ${ }^{[17]}$
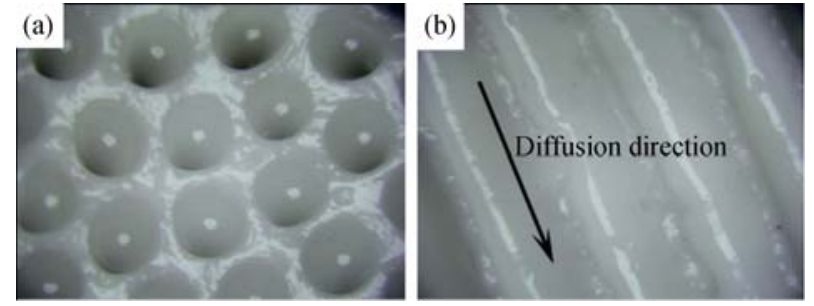

图 3 海藻酸钠 $/ \mathrm{Al}_{2} \mathrm{O}_{3}$ 凝胶的表面(a)和截面(b)的光学显微镜 照片

Fig. 3 Microstructures of surface (a) and cross-section (b) of $\mathrm{NaAlg} / \mathrm{Al}_{2} \mathrm{O}_{3}$ gels

在利用海藻酸钠制备多孔氧化铝陶瓷的过程中, 由于浆料中氧化铝的固相含量非常低 $(5 \mathrm{wt} \%$ $15 \mathrm{wt} \%)$, 所以干燥过程中会产生很大的收缩。如果 将离子凝胶后的湿坏直接置于空气中干燥，收缩率 达到 $60 \%$ 以上, 导致样品出现明显的裂纹, 这是由 于水在室温下表面张力非常高 $\left(72.8 \times 10^{-3} \mathrm{~N} / \mathrm{m}\right)$ 造成 的。所以, 本研究使用溶剂置换的方法, 将湿坏中的 水置换为叔丁醇，而叔丁醇的表面张力较低(19.5× $\left.10^{-3} \mathrm{~N} / \mathrm{m}\right)$ 。湿坏溶剂置换后, 分别采用冷冻干燥和 室温空气干燥两种方法进行干燥。图 4 为不同方法 干燥并烧结后样品的显微形貌。从图 4(a)中可以看 出, 冷冻干燥后的样品孔结构高度有序, 呈六方排 列，与图 3 中凝胶的孔结构保持一致。而如图 4(b) 所示, 空气中干燥的样品孔径大小不一, 形状杂乱, 与凝胶中的原始孔结构相差很大。所以, 利用冷冻 干燥法可以很好地控制材料在干燥过程中的收缩, 使显微结构保持完整。

图 5 为不同 $\mathrm{Al}_{2} \mathrm{O}_{3}$ 固相含量制备的直通孔多孔 陶瓷的 SEM 照片。从图 5 可以看出, 这三种固相含 量获得的样品都具有高度有序六方排列的孔结构, 说明 $\mathrm{Al}_{2} \mathrm{O}_{3}$ 固相含量在 $5 \mathrm{wt} \% \sim 15 \mathrm{wt} \%$ 范围内变化时 不会对海藻酸钠的离子凝胶过程和孔的形成过程产 生明显影响。当 $\mathrm{Al}_{2} \mathrm{O}_{3}$ 固相含量从 $5 \mathrm{wt} \%$ 提高到 $15 \mathrm{wt} \%$, 孔径大小维持在 $200 \mu \mathrm{m}$ 变化不大，而孔壁 厚度显著增大，说明更多的 $\mathrm{Al}_{2} \mathrm{O}_{3}$ 富集到了孔壁上。
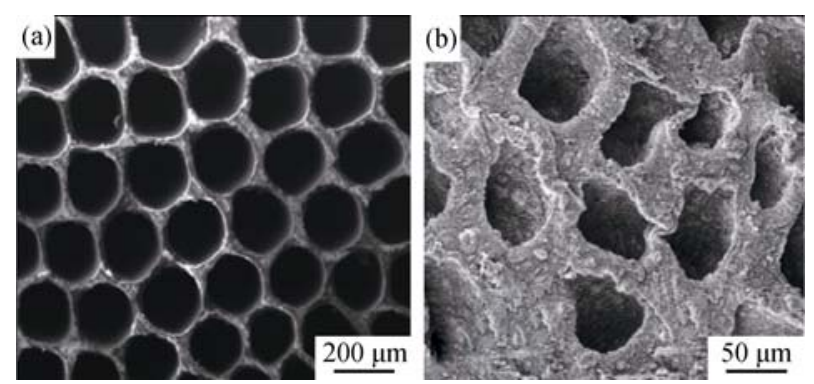

图 4 不同干燥方法对材料显微结构的影响

Fig. 4 Effects of drying methods on microstructures (a) Freeze-drying; (b) Evaporating in air 

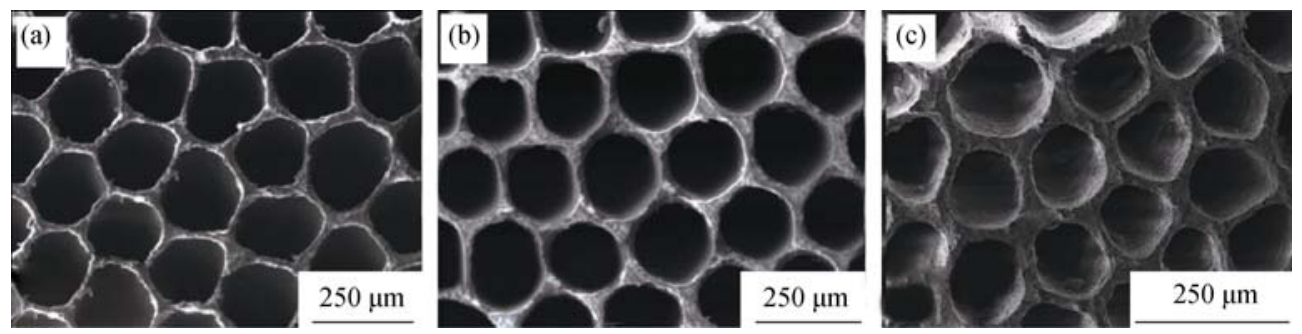

图 5 不同 $\mathrm{Al}_{2} \mathrm{O}_{3}$ 固相含量材料显微结构的 SEM 照片

Fig. 5 SEM images of microstructures with different solid loadings (a) $5 \mathrm{wt} \%$; (b) $10 \mathrm{wt} \%$; (c) $15 \mathrm{wt} \%$

为了观察 $\mathrm{Al}_{2} \mathrm{O}_{3}$ 在样品孔壁上的存在状态, 实 验利用扫描电镜对多孔陶瓷的孔壁进行了观察, 结 果如图 6 所示。从图 6 可以看出, 烧结后 $\mathrm{Al}_{2} \mathrm{O}_{3}$ 颗 粒长大, 相互连接, 但是孔壁不是完全致密的, 依 然存在一些 $<1 \mu \mathrm{m}$ 微孔。

\section{3 孔径分布}

为了更好地对材料中的孔进行表征，实验利用 压录仪对材料的孔径分布进行了测试，如图 7 所示。 从图 7 可以看出, 多孔陶瓷的孔径呈双峰分布, 大 孔范围在 170 200 $\mu \mathrm{m}$, 对应的是样品中规则排列的 直通孔结构; 而小孔范围在 $0.3 \sim 0.5 \mu \mathrm{m}$, 对应的是 孔壁上存在的孔结构。随着固相含量提高, 大孔尺 寸略微减小，而当固相含量提高到 $10 \mathrm{wt} \%$ 以上，小 孔尺寸明显减小, 说明当固相含量提高到一定程度, 氧化铝在孔壁上的富集作用更加明显, 使得烧结后 孔壁的密度显著上升，孔径减小。

\section{4 密度、渗透率与抗压强度}

表 1 给出了不同固相含量样品的密度、气孔 率、渗透率和抗压强度。从表 1 中可以看出, 随 着固相含量的增大, 材料的密度从 $0.87 \mathrm{~g} / \mathrm{cm}^{3}$ 提 高到 $1.16 \mathrm{~g} / \mathrm{cm}^{3}$, 相应的开气孔率从 $70.5 \%$ 下降到 $63.5 \%$ 。这是由于固相含量增加使得更多的 $\mathrm{Al}_{2} \mathrm{O}_{3}$ 富集到孔壁上, 增大了孔壁的厚度, 这与显微结构

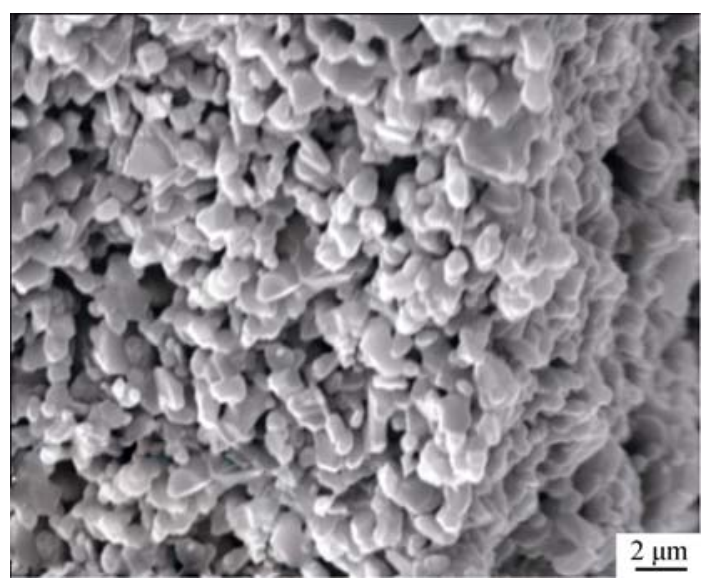

图 $6 \mathrm{Al}_{2} \mathrm{O}_{3}$ 多孔陶瓷孔壁显微结构 SEM 照片

Fig. 6 Microstructures of the pore walls of alumina porous ceramics

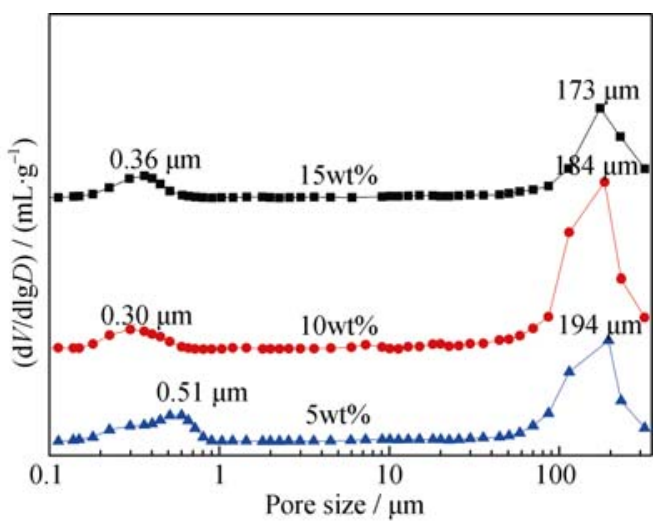

图 7 不同固相含量样品的孔径分布

Fig. 7 Pore size distribution of samples with different solid loadings

和气孔分布的结果相一致。随着固相含量的增加，材 料的渗透率也从 $2.57 \times 10^{-11} \mathrm{~m}^{2}$ 下降到 $2.16 \times 10^{-11} \mathrm{~m}^{2}$, 这是因为固相含量的升高使得直通孔孔径减小，孔 隙率也减小，从而降低了渗透率。从表 1 中还可以 看到，随着固相含量的增大，样品的抗压强度从 (18.9 \pm 3.2$) \mathrm{MPa}$ 提高到(44.2 \pm 5.4$) \mathrm{MPa}$ ，如此高的强 度得益于材料中定向、均匀排列的孔道结构 ${ }^{[18]}$ 。

\section{5 热导率}

表 2 给出了样品不同方向测得的热导率, 其中, 垂 直于孔道方向 $(\perp)$ 是指测试时热流的方向与孔道方向 垂直, 平行于孔道方向 (//) 是指测试时热流的方向与 孔道方向平行。由表 2 可以看出，垂直于孔道方向和平 行于孔道的室温热导率都随固相含量升高而增大，随

表 1 不同固相含量样品的密度、气孔率、渗透率和抗压强度

Table 1 Density, open porosity, permeability and compressive strength of samples with different solid loadings

\begin{tabular}{ccccc}
\hline $\begin{array}{c}\text { Solid } \\
\text { loading } \\
/ \mathrm{wt} \%\end{array}$ & $\begin{array}{c}\text { Open po- } \\
\text { rosity } \\
/ \%\end{array}$ & $\begin{array}{c}\text { Density/ } \\
\left(\mathrm{g} \cdot \mathrm{cm}^{-3}\right)\end{array}$ & $\begin{array}{c}\text { Compressive } \\
\text { strength } \\
/ \mathrm{MPa}\end{array}$ & $\begin{array}{c}\text { Permeability } \\
/\left(\times 10^{-11}, \mathrm{~m}^{2}\right)\end{array}$ \\
\hline 5 & 70.5 & 0.87 & $18.9 \pm 3.2$ & 2.57 \\
10 & 66.1 & 0.99 & $32.8 \pm 4.1$ & 2.42 \\
15 & 63.5 & 1.16 & $44.2 \pm 5.4$ & 2.16 \\
\hline
\end{tabular}


表 2 不同固相含量样品的室温热导率

Table 2 Thermal conductivity of samples with different solid loadings

\begin{tabular}{ccc}
\hline $\begin{array}{c}\text { Solid load- } \\
\text { ing } / \mathrm{wt} \%\end{array}$ & $\begin{array}{c}\text { Thermal conductiv- } \\
\text { ity } / /\left(\mathrm{W} \cdot \mathrm{m}^{-1} \cdot \mathrm{K}^{-1}\right)\end{array}$ & $\begin{array}{c}\text { Thermal conductiv- } \\
\text { ity }_{\perp} /\left(\mathrm{W} \cdot \mathrm{m}^{-1} \cdot \mathrm{K}^{-1}\right)\end{array}$ \\
\hline 5 & 2.08 & 1.36 \\
10 & 2.18 & 1.57 \\
15 & 3.03 & 1.78 \\
\hline
\end{tabular}

着固相含量从 $5 \mathrm{wt} \%$ 提高到 $15 \mathrm{wt} \%$, 平行孔道方向的热 导率从 $2.08 \mathrm{~W} /(\mathrm{m} \cdot \mathrm{K})$ 提高到 $3.03 \mathrm{~W} /(\mathrm{m} \cdot \mathrm{K})$, 而垂直孔道 方向的热导率从 $1.36 \mathrm{~W} /(\mathrm{m} \cdot \mathrm{K})$ 提高到 $1.78 \mathrm{~W} /(\mathrm{m} \cdot \mathrm{K})$, 并 且平行孔道方向热导率的增加幅度要明显大于垂直 孔道方向。这主要是因为固相含量升高, 样品的气 孔率降低, 从而样品中的连续相-氧化铝的热导率 主导作用越明显, 因此样品的热导率增大。

\section{3 结论}

1) 采用溶剂置换结合冷冻干燥的工艺, 利用海 藻酸钠离子的凝胶过程成功地制备了具有高度规则 六方排列直通孔结构的多孔氧化铝陶瓷，孔径大小 在 $200 \mu \mathrm{m}$ 左右，开气孔率在 $63.5 \%$ 70.5\%之间;

2) 通过调节固相含量可以对样品的孔径大小、分 布、孔隙率以及渗透率、抗压强度和热导率进行调控。

\section{参考文献:}

[1] DEVILLE S. Freeze-casting of porous ceramics: a review of current achievements and issues. Adv. Eng. Mater, 2008, 10(3): 155-169.

[2] MOON Y W, SHIN K H, KOH Y H, et al. Three-dimensional ceramic/camphene-based coextrusion for unidirectionally macrochanneled alumina ceramics with controlled porous walls. J. Am. Ceram. Soc., 2014, 97(1): 32-34.

[3] SOON Y M, SHI K H, KOH Y H, et al. Fabrication and compressive strength of porous hydroxyapatite scaffolds with a functionally graded core/shell structure. J. Euro. Ceram. Soc., 2011, 31(1/2): 13-15.

[4] CHOI H J, YANG T Y, YOON S Y, et al. Porous alumina/zirconia layered composites with unidirectional pore channels processed using a tertiary-butyl alcohol-based freeze casting. Mater. Chem. Phys., 2012, 133(1): 16-20.

[5] VOGLI E, SIEBER H AND GREIL P. Biomorphic SiC-ceramic prepared by Si-vapor phase infiltration of wood. J. Euro. Ceram. Soc., 2002, 22(14/15): 2663-2668.
[6] CHEN R F, WANG C A, HUANG Y, et al. Ceramics with special porous structures fabricated by freeze-gelcasting: using tert-butyl alcohol as a template. J. Am. Ceram. Soc., 2007, 90(11): 3478 3483 .

[7] CHEN R F, HUANG Y, WANG C A, et al. Ceramics with ultralow density fabricated by gelcasting: an unconventional view. $J$. Am. Ceram. Soc., 2007, 90(11): 3424-3430.

[8] ZHANG G J, YANG J F, OHJI T. Fabrication of porous ceramics with unidirectionally aligned continuous pores. J. Am. Ceram. Soc., 2001, 84(6): 1395-1397.

[9] PATERMARAKIS G, MOUSSOUTZANIS K. Transformation of porous structure of anodic alumina films formed during galvanostatic anodising of aluminium. J. Electroanal. Chem., 2011, 659(2): 176-190.

[10] ZHENG J Y, PANG J B, QIU K Y, et al. Synthesis of mesoporous silica materials with hydroxyacetic acid derivatives as templates via a Sol-Gel process. J. Inorg. Organomet. Polym., 2000, 10(3): 103-113.

[11] THIELE H. Histolyse und Histogenese: Gewebe und Ionotrope Gele-Prinzip einer Strukturbildung. Akademische Verlagsgesellschaft, Frankfurt, 1967.

[12] THUMBS J, KOHLER H H. Capillaries in alginate gel as an example of dissipative structure formation. Chem. Phys., 1996, 208(1): 9-24.

[13] DITTRICH R, TOMANDL G, DESPANG F, et al. Scaffolds for hard tissue engineering by ionotropic gelation of alginate-influence of selected preparation parameters. J. Am. Ceram. Soc., 2007, 90(6): 1703-1708.

[14] BULUT E, SANLI O. Optimization of release conditions of alzheimer's drug donepezil hydrochloride from sodium Alginate/ Sodium carboxymethyl cellulose blend microspheres. J. Macro. Sci. $B, 2014$, 53(5): 902-917.

[15] FENG C, SONG R X, SUN G H, et al. Immobilization of coacervate microcapsules in multilayer sodium alginate beads for efficient oral anticancer drug delivery. Biomacromolecules, 2014, 15(3): 985-996.

[16] FUKUSHIMA M, NAKATA M, ZHOU Y, et al. Fabrication and properties of ultra highly porous silicon carbide by the gelationfreezing method. J. Eur. Ceram. Soc., 2010, 30(14): 2889-2896.

[17] ELJAOUHARI A A, MULLER R, KELLERMEIER M, et al. New anistropic ceramic membranes from chemically fixed dissipative structures. Langmuir, 2006, 22:11353-11359.

[18] DEVILLE S. Freeze-casting of porous biomaterials: structure, properties and opportunities. Materials, 2010, 3(3): 1913-1927. 